\title{
CAPACITÉS ADAPTATIVES DU TURBOT (PSETTA MAXIMA) JUVÉNILE À LA PHOTOPÉRIODE.
}

\author{
K. PICHAVANT, J. PERSON-Le RUYeT, A. SEVERE, A. Le ROUX, L. QUEMENER \\ et G. BOEUF
}

IFREMER, Laboratoire de Physiologie des Poissons, BP 70, 29280 Plouzané, France.

\section{RÉSUMÉ}

Des turbots d'un poids moyen initial de $32 \mathrm{~g}$ ont été exposés pendant 60 jours à 4 photopériodes constantes (8L:16O, 12L:12O, 16L:8O, 24L:0O) et à 2 photophases variables : l'une croissante (de 12 à $16 \mathrm{~h}$ ) et l'autre décroissante (de 12 à $8 \mathrm{~h}$ ). Les autres paramètres du milieu ont été maintenus constants pendant les 2 mois de l'expérimentation (température : $17 \pm 0,5^{\circ} \mathrm{C}$, salinité : $34,5 \mathrm{ppm}$, intensité lumineuse : $2 \mathrm{~W} \cdot \mathrm{m}^{-2}$, concentration

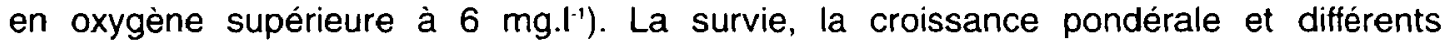
indicateurs alimentaires (taux de conversion apparent, coefficients d'utilisation et d'efficacité protéiques) ont été déterminés. L'excrétion azotée (niveaux journaliers et profils horaires de l'excrétion d'Azote Ammoniacal Total, AAT, et d'azote uréique) et certains paramètres plasmatiques (osmolarité, natrémie, kaliémie, chlorémie, niveaux circulants des hormones thyroïdiennes) ont été mesurés.

Au cours de l'expérience, aucune mortalité n'est apparue. Les croissances pondérales et les taux de croissance spécifique ont été identiques pour tous les traitements (poids moyens multipliés par trois en 60 jours). De même, la prise alimentaire $(1,5-1,7 \%$ de la biomasse par jour), le taux de conversion alimentaire $(0,70-0,75)$ et les coefficients d'utilisation (36-39\%) et d'efficacité $(2,5-2,7)$ protéiques ne sont pas influencés par la photopériode. La composition corporelle des animaux en protéines, lipides totaux, cendres et eau n'a pas subi de modification au cours de l'expérimentation. Osmolarité et électrolytes plasmatiques sont stables et identiques pour les différentes conditions. La thyroxine est stable quelle que soit la photopériode, les niveaux circulants sont compris entre 2 et 4 ng.ml'. Les concentrations plasmatiques en $T_{3}$ apparaissent significativement différentes en fin d'expérience : elles sont plus basses chez les animaux en photophase décroissante et chez ceux maintenus en photophase courte $(8 \mathrm{~h})$. Les taux journaliers d'excrétion azotée sous la forme d'AAT (200-230 mg.kg $\left.{ }^{-} \cdot \mathrm{j}^{-1}\right)$ et d'urée (65-75 mg.kg-1..j) sont identiques pour tous les traitements. Les profils d'excrétion azotée ont présenté des différences notables selon la photopériode. L'excrétion d'AAT est caractérisée par un pic post-prandial chez les animaux soumis à une alternance jour/nuit. Par contre, en éclairage continu, les taux horaires d'excrétion d'AAT restent stables tout au long du nycthémère. L'urée présente un pic nocturne dont l'amplitude, l'étalement et l'heure d'apparition dépendent de la photopériode. En éclairage continu, cette augmentation de l'excrétion d'urée est observée à un moment durant lequel les autres poissons sont en phase nocturne.

A l'instar d'autres espèces de poissons plats, le turbot est peu sensible à la photopériode du moins lorsque les conditions d'alimentation sont ajustées à la durée de la phase éclairée.

Mots-clés : turbot, Psetta maxima, photopériode, croissance, excrétion, hormones thyroïdiennes. 


\title{
EFFECTS OF PHOTOPERIOD ON JUVENILE TURBOT (PSETTA MAXIMA) : PHYSIOLOGICAL STATUS AND GROWTH.
}

\begin{abstract}
Turbot juveniles (32 $\mathrm{g}$ of initial weight) have been reared for 60 days under different artificial photoperiods : four constant photoperiod regimes (8L:16D, 12L:12D, 16L:8D, $24 \mathrm{~L}: \mathrm{OD}$ ) and two changing regimes, one increasing from 12 to $16 \mathrm{~h}$ light per day, and the other decreasing from 12 to $8 \mathrm{~h}$ light per day. All other environmental conditions were maintained constant : temperature : $17 \pm 0.5^{\circ} \mathrm{C}$, salinity : $34.5 \mathrm{ppt}$, light intensity : $2 \mathrm{~W} \cdot \mathrm{m}^{2}$, $\mathrm{O}_{2}$ over $6 \mathrm{mg} . \mathrm{l}^{\prime}$. The effects of these photoperiods on growth, feed efficiency, nitrogenous excretion, physiological status (blood plasma osmolarity, chloride, sodium, potassium and thyroid hormones contents) were studied.
\end{abstract}

All over the experiment, fish looked healthy and no mortality was observed. No significant differences in growth response to photoperiod were observed. Specific growth rate $(1.7-1.9)$, apparent food conversion ratio $(0.70-0.75)$, protein efficiency ratio $(2.5-2.7)$ and protein utilization coefficient (36-39\%) were not photoperiod-dependent. There were no changes in fish body composition in terms of proteins, lipids, ash and water contents. No significant differences in blood plasma osmolarity, chloride, sodium and potassium concentrations, related to photoperiods, were observed. $T_{4}$ concentrations were similar for all photoperiods tested, they ranged from 2 to $4 \mathrm{ng}^{-\mathrm{ml}^{-1}}$ while $\mathrm{T}_{3}$ levels significantly decreased at the end of experiment in turbot maintained under the shortest day-lengths (8 hours light and decreasing photophase). A major post-prandial increase in total ammonia nitrogen excretion was observed under photophases $8 \mathrm{~h}, 12 \mathrm{~h}$ and $16 \mathrm{~h}$. Under permanent lighting, this peak was significantly lower. Urea nitrogen excretion showed a nocturnal peak related to photoperiod. In fish submitted to permanent lighting, the same urea pattern was observed.

In the Atlantic strain of turbot tested, growth and feed efficiency were not influenced by photoperiod at least within 60 days.

Key-words : turbot, Psetta maxima, photoperiod, growth, nitrogen excretion, thyroid hormones.

\section{INTRODUCTION}

Le développement et la croissance des poissons téléostéens se déroulent selon des étapes caractéristiques de l'espèce et sous la dépendance plus ou moins directe de facteurs internes (nerveux, endocriniens et neuroendocriniens) et externes. Ces derniers contrôlent ou synchronisent, plus ou moins étroitement, diverses activités ou fonctions du poisson dont la capacité à grandir. Nous pouvons ainsi définir des facteurs dits " déterminants " (ou " décisifs "), car ils exercent un effet direct sur certains " récepteurs " de l'animal qui se traduit ensuite par une augmentation ou une diminution de la croissance (température, salinité, lumière), et d'autres dits " limitants " car ils doivent soit rester en dessous (ammoniac), soit dépasser (oxygène) certains seuils dans le milieu. L'un des buts essentiels aujourd'hui en aquaculture est d'obtenir des animaux de qualité dans des conditions économiques acceptables. Ceci demande de judicieusement utiliser les facteurs du milieu afin de pouvoir mettre sur le marché des produits de qualité stable durant la plus grande période de l'année. 
Le turbot représente une espèce de poisson plat répandue dans la zone ouesteuropéenne et phylogéniquement très évoluée chez les téléostéens. II fait l'objet d'un développement aquacole depuis une dizaine d'années. Quelques travaux antérieurs à 1995 existaient dans la littérature sur les effets de divers facteurs " déterminants " de l'environnement sur le développement, la croissance et la survie de cette espèce. Nous pouvons citer entre autres les données de KÜHLMANN et QUANTZ (1980), WALLER (1992) sur la salinité ; JONES et al. (1981), IGLESIAS et al. (1987), PERSON-Le RUYET et al. (1991) sur la température et IMSLAND et al. (1995) sur la photopériode. Les travaux prenant réellement en compte les relations entre les données zootechniques et les réponses physiologiques du turbot, estimées par l'utilisation de divers indicateurs spécifiques, sont relativement récents : travaux de BUREL et al. (1996) sur la température et de GAUMET et al. (1995) sur la salinité. Afin de déterminer les conditions ambiantes optimales au " confort physiologique " et à la meilleure expression possible de la croissance de cette espèce, l'impact de la photopériode restait à préciser.

Des juvéniles de turbot d'un poids initial moyen de $32 \mathrm{~g}$ ont donc été exposés pendant 2 mois à différentes conditions photopériodiques. Les paramètres classiques indispensables en zootechnie (survie, évolution du poids, différents indicateurs de l'efficacité alimentaire) ont été mesurés. De plus, certaines réactions physiologiques, en relation avec les photopériodes appliquées, ont été étudiées : l'excrétion azotée (azote ammoniacal total et uréique), l'osmolarité, certains électrolytes du plasma sanguin $\left(\mathrm{Na}^{+}, \mathrm{K}^{+}\right.$, $\mathrm{Cl}$ ) et les niveaux circulants des hormones thyroïdiennes (tri-iodothyronine ou $T_{3}$ et thyroxine ou $\mathrm{T}_{4}$ ).

\section{MATÉRIEL ET MÉTHODES}

Les turbots utilisés pour l'étude étaient des juvéniles âgés de 6 mois d'un poids moyen initial de $32 \pm 3 \mathrm{~g}$ produits dans les structures expérimentales de l'IFREMER à Brest, selon les techniques proposées par PERSON-Le RUYET et al. (1991). Les animaux étaient stabulés dans des bassins de type suédois de $1 \mathrm{~m}^{2}$, d'un volume effectif de $400 \mathrm{I}$, alimentés par une eau filtrée et désaturée sur la base d'1,4 renouvellement. $h^{-1}$. L'intensité lumineuse était fixée à $2 \mathrm{~W} \cdot \mathrm{m}^{-2}$ à la surface de l'eau et l'oxygène disponible était maintenu au-dessus de $6 \mathrm{mg} . \mathrm{l}^{-1}$, le niveau post-prandial étant pris comme référence. Les poissons étaient nourris avec un aliment expansé coulant de type SEVBAR contenant $55 \%$ de protéines et $12 \%$ de lipides. II était enrichi en huile de foie de morue, lécithine de soja et mélange vitaminique avant consommation.

Les turbots ont été “ acclimatés " pendant 4 semaines à une photopériode 12L:12O et aux conditions d'alimentation et d'environnement choisies pour l'expérimentation : 34,5-35,5 ppm de salinité, température : $17 \pm 0,5{ }^{\circ} \mathrm{C}$. Puis, ils ont été soumis à six conditions photopériodiques durant 60 jours : 4 photopériodes constantes (8L:160, $12 \mathrm{~L}: 120,16 \mathrm{~L}: 80,24 \mathrm{~L}: 00$ ) et 2 photophases variables, une photophase croissante de 12 à $16 \mathrm{~h}$ (PPC) et une photophase décroissante de 12 à $8 \mathrm{~h}$ (PPD). Toutes ces conditions étaient réalisées en duplicat ( 2 bassins par condition). L'aliment a été distribué grâce à un distributeur automatique "calé "sur la longueur de la photophase moins deux heures (l'une en début, l'autre en fin de phase éclairée). Deux heures d'arrêt de l'alimentation étaient également respectées pour les animaux en lumière constante. La ration journalière distribuée a été ajustée à l'appétit des poissons grâce à l'évaluation, 3 fois par semaine, de la quantité d'aliment réellement ingérée. L'heure de début du repas était fixée à $9 \mathrm{~h}$ du matin pour toutes les conditions expérimentales. 
Un échantillonnage de 30 poissons par bassin (60 par condition) était pesé toutes les deux semaines après une mise à jeun de $24 \mathrm{~h}$. Juste avant la pesée, des prélèvements de sang étaient réalisés au niveau de l'aorte postérieure sur 6 individus par bassin (12 par condition). Le plasma était récupéré après centrifugation (3 $500 \mathrm{t} \cdot \mathrm{mn}^{-1}$ durant $10 \mathrm{mn}$ à $4{ }^{\circ} \mathrm{C}$ ) et stocké à $-28{ }^{\circ} \mathrm{C}$ jusqu'aux dosages ultérieurs. Les techniques de mesure des valeurs d'osmolarité, des ions et des hormones thyroïdiennes plasmatiques ont été présentées dans BOEUF et al. (1989) et MARTINEZ et al. (1995).

Des mesures de l'excrétion azotée ammoniacale et uréique ont été effectuées pour les 4 photopériodes constantes selon les méthodes adaptées de DOSDAT et al. (1995a) et décrites par BUREL et al. (1996). Trois profils horaires d'excrétion ont été réalisés sur une période de 26 heures à partir de prélèvements manuels d'un échantillon d'eau de mer toutes les heures. Simultanément, les taux d'excrétion journaliers ont été déterminés par collecte d'eau sur 24 heures grâce à une pompe péristaltique. Les dosages sont effectués à l'aide d'une chaine d'analyse en flux continu (Technicon) selon les méthodes décrites par DOSDAT et al. (1995a).

Différents indicateurs alimentaires ont pu être calculés : Taux de Conversion Apparent $\left(T C A=1 /\left(B_{2}-B_{1}\right)\right)$, Coefficients d'Efficacité $\left(C E P=\left(B_{2}-B_{1}\right) / P_{1}\right)$ et d'Utilisation (CUP $\left.=\left(P_{1} / P_{1}\right)^{\star} 100\right)$ Protéiques où 1 représente la quantité d'aliment ingéré au cours de l'expérimentation, B2 la biomasse finale, B1 la biomasse initiale, $\mathrm{Pi}$ la quantité de protéines ingérées et $\mathrm{Pf}$ la quantité de protéines fixées par les animaux. Des analyses biochimiques de la teneur en protéines, lipides, cendres et eau des poissons entiers ont été réalisées en début et fin d'expérimentation (ANONYME, 1984).

Les analyses statistiques ont été effectuées grâce au logiciel Statgraphics. Les données sont représentées par la moyenne suivie de l'erreur standard et le nombre d'échantillons ( $n$ ) est précisé. Si les valeurs obtenues pour les différents paramètres mesurés sont homogènes (homogénéité des variances vérifiée par le test de Bartlett, $p>0,05)$, elles sont comparées par une analyse de variance à une voie. En cas de différence significative $(p<0,05)$, un test de comparaison multiple de Tukey est appliqué. Lorsque les variances ne sont pas homogènes, un test de Kruskall-Wallis suivi, en cas de différence significative, d'un test de Students-Neumann-Keuls est utilisé.

\section{RÉSULTATS}

Durant les 2 mois d'expérimentation, aucune mortalité n'a été observée. Les résultats des différents paramètres zootechniques et physiologiques étant identiques pour les réplicats, les valeurs ont été regroupées pour chaque condition photopériodique. Aucun effet significatif de la photopériode n'apparaît sur la croissance pondérale (Figure 1) et les Taux de Croissance Spécifique (TCS, Tableau I). Les poids moyens sont multipliés par trois au cours des 60 jours d'expérimentation: ils évoluent de $32 \pm 3 \mathrm{~g}$ à $95 \pm 3 \mathrm{~g}$ en fin d'expérimentation, toutes photopériodes confondues. Le taux de conversion apparent $(0,7-0,75)$, les coefficients d'utilisation (36-39\%) et d'efficacité protéiques $(2,5-2,7)$ sont similaires quelles que soient les conditions photopériodiques (Tableau I). La prise alimentaire a diminué progressivement au cours de l'expérience en relation avec l'augmentation du poids des animaux mais elle n'a pas été influencée par la photopériode : elle a ainsi évolué de $1,7 \%$ de la biomasse.jour ${ }^{\prime}$ de $J_{0}$ à $J_{30}$ à $1,5 \%$ de la biomasse.jour' de $J_{30}$ à $J_{60}$. Aucune modification significative de la composition biochimique en termes de protéines, lipides totaux, cendres et eau des poissons entiers n'a été observée en fonction des conditions expérimentales (Tableau II). 


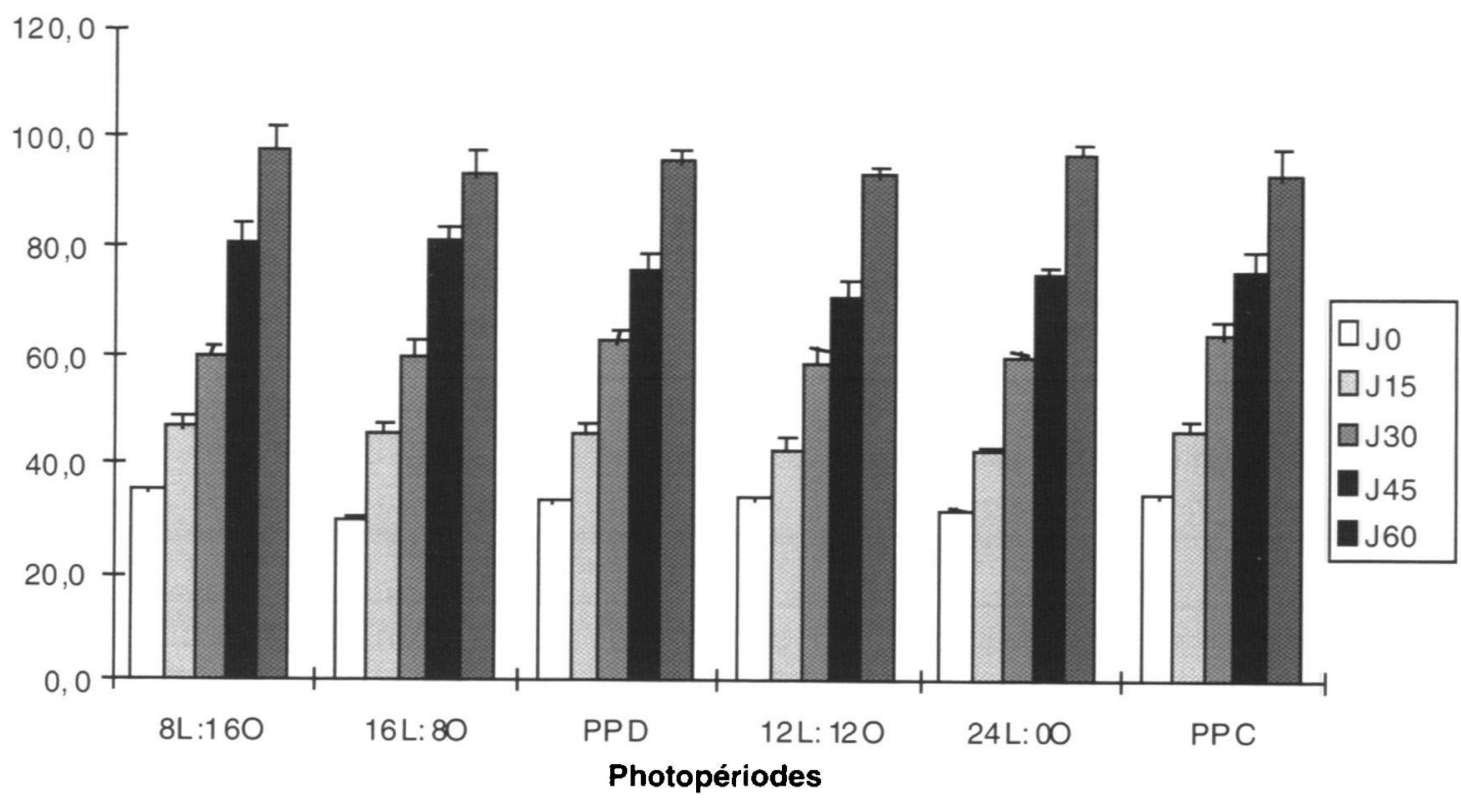

Figure 1

Evolution des poids moyens individuels de juvéniles de turbot pour les 6 photopériodes expérimentales en fonction des dates d'échantillonage : J0, J15, $\mathrm{J} 30, \mathrm{~J} 45$ et J60. Les moyennes sont représentées avec l'erreur standard $(n=60)$. L'absence de lettre indique l'absence de différence significative.

Figure 1

Changes over time (Days $0,15,30,45$ and 60 of the experiment) of mean weight in relation with photoperiod. Results are expressed as mean \pm standard error $(n=60)$. No superscripted letters indicate no statistical differences.

\section{Tableau I}

Taux de Croissance Spécifique (TCS), Taux de Conversion Alimentaire (TCA), Coefficient d'Efficacité (CEP) et d'Utilisation (CUP) Protéique en fonction des conditions photopériodiques sur une période de 60 jours. Les moyennes sont représentées avec l'erreur standard $(n=2)$. L'absence de lettre indique l'absence de différence significative.

\section{Table I}

Specific growth rate, apparent food conversion, protein efficiency ratio and protein utilization coefficient related to the different photoperiods for 60 days. Results are expressed as mean \pm standard error $(n=2)$. No superscripted letters indicate no statistical differences.

PHOTOPÉRIODES

\begin{tabular}{l|cccccc}
\hline & $8 L: 16 D$ & $12 L: 12 D$ & $16 L: 8 D$ & $24 L: 0 D$ & PPC & PPD \\
\cline { 2 - 7 } TCS & $1,8 \pm 0,1$ & $1,8 \pm 0,1$ & $1,8 \pm 0,1$ & $1,9 \pm 0,1$ & $1,8 \pm 0,1$ & $1,7 \pm 0,1$ \\
TCA & $0,73 \pm 0,02$ & $0,70 \pm 0,02$ & $0,72 \pm 0,03$ & $0,71 \pm 0,05$ & $0,68 \pm 0,01$ & $0,71 \pm 0,02$ \\
CEP & $2,50 \pm 0,07$ & $2,68 \pm 0,19$ & $2,63 \pm 0,1$ & $2,71 \pm 0,10$ & $2,67 \pm 0,07$ & $2,54 \pm 0,13$ \\
CUP & $37,5 \pm 1,0$ & $37,7 \pm 2,8$ & $38,4 \pm 1,4$ & $39,8 \pm 0,1$ & $36,8 \pm 1,0$ & $37,4 \pm 1,9$ \\
\hline
\end{tabular}


Tableau II

Composition en eau, cendres, lipides et protéines des poissons à J60 de l'expérimentation en fonction des photopériodes, en $\%$ du poids frais des animaux. Les moyennes sont représentées avec l'erreur standard $(n=9)$. L'absence de lettre indique l'absence de différence significative.

Table II

Final body composition (water, ash, lipids and proteins contents) of turbot acclimated to different photoperiods for 60 days. Means are given with standard error $(n=9)$ and are expressed as percentage of body weight. No superscripted letters indicate no statistical differences.

\begin{tabular}{c|cccccc}
\hline & \multicolumn{7}{c}{ PHOTOPÉRIODES } \\
\hline & $8 \mathrm{~L}: 16 \mathrm{D}$ & $12 \mathrm{~L}: 12 \mathrm{D}$ & $16 \mathrm{~L}: 8 \mathrm{D}$ & $24 \mathrm{~L}: 0 \mathrm{D}$ & PPC & PPD \\
\cline { 2 - 7 } Protéines (\%) & $15,0 \pm 0,1$ & $14,6 \pm 0,2$ & $14,6 \pm 0,2$ & $14,7 \pm 0,2$ & $14,3 \pm 0,3$ & $14,3 \pm 0,3$ \\
Lipides (\%) & $3,0 \pm 0,2$ & $2,8 \pm 0,2$ & $3,2 \pm 0,1$ & $3,2 \pm 0,1$ & $2,7 \pm 0,1$ & $2,7 \pm 0,1$ \\
Cendres (\%) & $3,5 \pm 0,2$ & $3,3 \pm 0,1$ & $3,3 \pm 0,1$ & $3,1 \pm 0,2$ & $3,3 \pm 0,4$ & $3,3 \pm 0,1$ \\
& $76,8 \pm 0,3$ & $77,6 \pm 0,3$ & $77,1 \pm 0,1$ & $77,1 \pm 0,2$ & $77,9 \pm 0,2$ & $77,9 \pm 0,2$ \\
\hline
\end{tabular}

D'autre part, aucun effet de la photopériode n'a été mis en évidence sur les différents paramètres physiologiques mesurés. Osmolarité et électrolytes plasmatiques sont stables et identiques entre les différentes conditions (Tableau III). La thyroxine est stable pour toutes les photopériodes et les niveaux circulants sont compris entre 2 et $4 \mathrm{ng} \cdot \mathrm{ml}^{-1}$ (Tableau IVa). Les concentrations plasmatiques en $T_{3}$ sont significativement différentes en fin d'expérimentation: elles sont plus faibles chez les animaux en photophase décroissante et chez ceux maintenus en photophase courte $(8 \mathrm{~h})$ (Tableau IVb).

\section{Tableau III}

Osmolarité, concentrations plasmatiques en $\mathrm{Cl}^{-}, \mathrm{Na}^{+}$et $\mathrm{K}^{+}$moyennées de $\mathrm{J0}$ à $\mathrm{J} 60$ pour les 6 conditions photopériodiques. Les moyennes sont exprimées avec l'erreur standard $(n=60)$. L'absence de lettre indique l'absence de différence significative.

Table III

Plasma osmolarity, $\mathrm{Cl}^{-}, \mathrm{Na}^{+}$and $\mathrm{K}^{+}$concentrations in turbot exposed to 6 different photoperiods for 60 days. Means are given with standard error $(n=60)$. No superscripted letters indicate no statistical differences.

\begin{tabular}{|c|c|c|c|c|c|c|}
\hline \multirow[b]{3}{*}{$\begin{array}{l}\text { Osmolarité } \\
\text { mOsmol.l" }\end{array}$} & \multicolumn{6}{|c|}{ PHOTOPÉRIODES } \\
\hline & $8 \mathrm{~L}: 16 \mathrm{D}$ & $12 \mathrm{~L}: 12 \mathrm{D}$ & $16 \mathrm{~L}: 8 \mathrm{D}$ & 24L:OD & PPC & PPD \\
\hline & $327 \pm 2$ & $330 \pm 1$ & $326 \pm 2$ & $332 \pm 2$ & $328 \pm 1$ & $325 \pm 3$ \\
\hline$[\mathrm{Cl}] \mathrm{mM}$ & $148 \pm 2$ & $149 \pm 1$ & $147 \pm 3$ & $151 \pm 3$ & $148 \pm 2$ & $147 \pm 1$ \\
\hline$\left[\mathrm{Na}^{+}\right] \mathrm{mM}$ & $170 \pm 1$ & $177 \pm 2$ & $172 \pm 2$ & $170 \pm 3$ & $172 \pm 3$ & $169 \pm 4$ \\
\hline$\left[\mathrm{K}^{+}\right] \mathrm{mM}$ & $2,2 \pm 0,8$ & $3.1 \pm 0,5$ & $2,4 \pm 0,7$ & $3.0 \pm 0.4$ & $3,2 \pm 0,3$ & $2,6 \pm 0,5$ \\
\hline
\end{tabular}




\section{Tableau IVa}

Concentrations plasmatiques en $T_{4}$ pour les 6 photopériodes expérimentales entre les jours 0 et 60 de l'expérience. Les moyennes sont représentées avec l'erreur standard ( $n=12$ pour chaque date d'échantillonage). L'absence de lettre exprime une homogénéité globale.

Table IVa

Plasma $\mathrm{T}_{4}$ concentrations on day $0,15,30,45$ and 60 of the experiment in turbots exposed to 6 different photoperiods. Means are given with standard error $(n=12)$. No superscripted letters indicate no statistical differences.

\begin{tabular}{c|cccccc}
\hline \multicolumn{7}{c}{ PHOTOPÉRIODES } \\
\hline Temps & 8L:16D & $12 \mathrm{~L}: 12 \mathrm{D}$ & $16 \mathrm{~L}: 8 \mathrm{D}$ & $24 \mathrm{~L}: 0 \mathrm{D}$ & $\mathrm{PPC}$ & PPD \\
\hline J0 & \multicolumn{7}{c}{$3,3 \pm 0,2$} \\
\hline $\mathrm{J15}$ & $2,4 \pm 0,7$ & $2,6 \pm 0,4$ & $2,4 \pm 0,3$ & $2,4 \pm 0,3$ & $2,5 \pm 0,6$ & $3,3 \pm 0,4$ \\
$\mathrm{~J} 30$ & $3,1 \pm 0,3$ & $2,5 \pm 0,3$ & $2,2 \pm 0,7$ & $1,7 \pm 0,8$ & $2,0 \pm 0,5$ & $2,5 \pm 0,6$ \\
$\mathrm{~J} 45$ & $2,5 \pm 0,6$ & $2,1 \pm 0,7$ & $2,3 \pm 0,9$ & $1,7 \pm 0,5$ & $2,2 \pm 0,5$ & $2,9 \pm 0,4$ \\
$\mathrm{~J} 60$ & $3,1 \pm 0,8$ & $3,5 \pm 0,4$ & $4,5 \pm 0,6$ & $3,4 \pm 1,0$ & $4,6 \pm 0,9$ & $3,1 \pm 0,5$ \\
\hline
\end{tabular}

\section{Tableau IVb}

Evolution des concentrations plasmatiques en $T_{3}$ pour les 6 photopériodes expérimentales entre les jours 0 et 60 de l'expérience. Les moyennes sont représentées avec l'erreur standard ( $n=12$ pour chaque date d'échantillonnage) et lorsqu'elles sont accompagnées d'une lettre commune, elles ne sont pas significativement différentes. L'absence de lettre exprime une homogénéité globale.

\section{Table IVb}

Plasma $T_{3}$ concentrations on day $0,15,30,45$ and 60 of the experiment in turbots exposed to 6 photoperiods. Means are given with standard error $(n=12)$. Superscripted letters indicate intergroup statistical differences per photoperiod; means not sharing with a common letter are significantly different.

\begin{tabular}{|c|c|c|c|c|c|c|}
\hline \multirow[b]{2}{*}{ Temps } & \multicolumn{6}{|c|}{ PHOTOPÉRIODES } \\
\hline & $8 \mathrm{~L}: 16 \mathrm{D}$ & $12 \mathrm{~L}: 12 \mathrm{D}$ & 16L:8D & 24L:OD & PPC & PPD \\
\hline Jo & \multicolumn{6}{|c|}{$2,16 \pm 0,2$} \\
\hline $\mathrm{J} 15$ & $2,1 \pm 0,3$ & $3,0 \pm 0,5$ & $2,16 \pm 0,1$ & $2,3 \pm 0,5$ & $2,0 \pm 0,1$ & $2,5 \pm 0,4$ \\
\hline $\mathrm{J} 30$ & $2,9 \pm 0,5$ & $3,5 \pm 0,5$ & $3,0 \pm 0,3$ & $2,8 \pm 0,2$ & $3,43 \pm 0,2$ & $3,8 \pm 0,2$ \\
\hline $\mathrm{J} 45$ & $3,4 \pm 0,4$ & $2,4 \pm 0,2$ & $2,3 \pm 0,2$ & $2,7 \pm 0,4$ & $2,2 \pm 0,4$ & $2,8 \pm 0,1$ \\
\hline $\mathrm{J} 60$ & $2,9 \pm 0,3^{\circ}$ & $4,6 \pm 0,3^{\circ}$ & $4,4 \pm 0,4^{\circ}$ & $4,9^{\prime} \pm 0,3^{\circ}$ & $4,9 \pm 0,3^{\circ}$ & $3,3 \pm 0,6^{a}$ \\
\hline
\end{tabular}


Les taux journaliers d'excrétion azotée sont identiques pour toutes les conditions expérimentales : 200 à $230 \mathrm{mg}$ d'AAT excrété. $\mathrm{kg}^{-1} .^{-j^{-1}}$ et 65 à $75 \mathrm{mg}$ d'urée excrétée. $\mathrm{kg}^{\mathrm{i}} \cdot \mathrm{j}^{-1}$. L'AAT est la principale forme d'excrétion azotée et représente 74 à $76 \%$ de l'excrétion azotée totale mesurée (AAT + urée) pour tous les traitements. Les profils d'excrétion azotée présentent des différences notables. L'excrétion d'AAT (Figure 2a) montre un pic post-prandial pour les animaux exposés à une alternance jour/nuit. Une augmentation des taux horaires d'excrétion d'AAT est ainsi observée une heure après le début du repas, le maximum étant obtenu environ 6 heures après la distribution de l'aliment : ils varient de 2 (niveau de base) à 18 (maximum) mg d'AAT excrété. $\mathrm{kg}^{-1} \cdot \mathrm{h}^{-1}$. Le retour au niveau de base est progressif, il est atteint vers 6-7 h du matin soit environ 21 heures après le début de l'alimentation. Pour les animaux acclimatés à une photophase continue et alimentés sur une période de 22 heures, les taux horaires d'excrétion restent relativement stables tout au long du nycthémère et sont compris entre 6 et $12 \mathrm{mg}$ d'AAT excrété $\mathrm{kg}^{-1} \cdot \mathrm{h}^{-1}$. Le profil d'excrétion uréique est fondamentalement différent (Figure $2 \mathrm{~b}$ ). II se caractérise par un pic nocturne dont l'amplitude, l'étalement et l'heure d'apparition dépendent de la photopériode. L'amplitude maximale du pic d'excrétion uréique est ainsi observée au milieu de la scotophase pour les photopériodes 8L:160,12L:12O et 16L:80. Pour ces conditions, les taux horaires d'excrétion uréique varient entre 2 (niveau de base) et 17 (maximum) $\mathrm{mg}$ d'urée excrétée. $\mathrm{kg}^{-1} \cdot \mathrm{h}^{-1}$. En éclairage continu, les poissons montrent également une augmentation de l'excrétion d'urée à un moment correspondant à la phase nocturne chez les autres lots. Toutefois, le pic d'excrétion est de moindre amplitude (maximum $10 \mathrm{mg}$ d'urée excrétée. $\mathrm{kg}^{-1} \cdot \mathrm{h}^{-1}$ ).

\section{DISCUSSION}

Dans le cadre d'expériences, menées antérieurement dans notre laboratoire, avec une souche de turbot du proche-Atlantique stabulée depuis quelques années au centre IFREMER de Brest, les limites de son " confort physiologique " ont été précisées en faisant varier la salinité (GAUMET et al., 1995) et la température (BUREL et al., 1996). Cette expérience sur la photopériode, menée dans les mêmes conditions, permet de compléter ces travaux.

La photopériode, dans la plage étudiée (photophase de 8 à 24 heures), pour la souche utilisée, et dans les conditions d'alimentation retenues (même ration mais étalement sur la longueur de la phase éclairée moins deux heures), n'a aucun effet significatif sur la croissance des turbots pendant les 60 jours d'expérimentation. Dans une étude sur la même espèce, IMSLAND et al. (1995) avaient obtenu un léger effet positif des photophases longues sur la capacité à grandir lors du dernier échantillonnage effectué sur des animaux de $8 \mathrm{~g}$ de poids initial, après 180 jours de stabulation sous 3 photopériodes (naturelle, 16L:80 et 24L:0O). De même, chez d'autres espèces de poissons plats marins, la sole, Solea solea, la plie, Pleuronectes platessa, le flétan, Hippoglossus hippoglossus, la photopériode n'a pas d'impact majeur sur la capacité à grandir des animaux : soit elle n'a pas d'effet, soit celui-ci n'apparaît qu'après une très longue période de temps et demeure de toute façon très limité (FONDS, 1979 ; HALLARÅKER et al., 1995). Cependant, chez d'autres espèces de poissons marins telles que le sébaste, Sebastes diploproa (BOEHLERT, 1981), la daurade, Sparus auratus (SILVA-GARCIA, 1996) ou encore Lepomis cyanellus (GROSS et al., 1965), la longueur du jour peut s'avérer influer sur la croissance (BOEUF et LE BAIL, sous presse). Toutefois même chez ces espèces, les effets de la photopériode sont beaucoup moins prononcés que chez les espèces de poissons anadromes. Chez le saumon atlantique, Salmo salar, par exemple, les effets de très longues photophases peuvent être spectaculaires, tant en eau douce (accélération de la croissance et de la smoltification) que lors du grossissement en eau de mer (SAUNDERS et al., 1989 ; FORSBERG, 1995). 
25

(a)

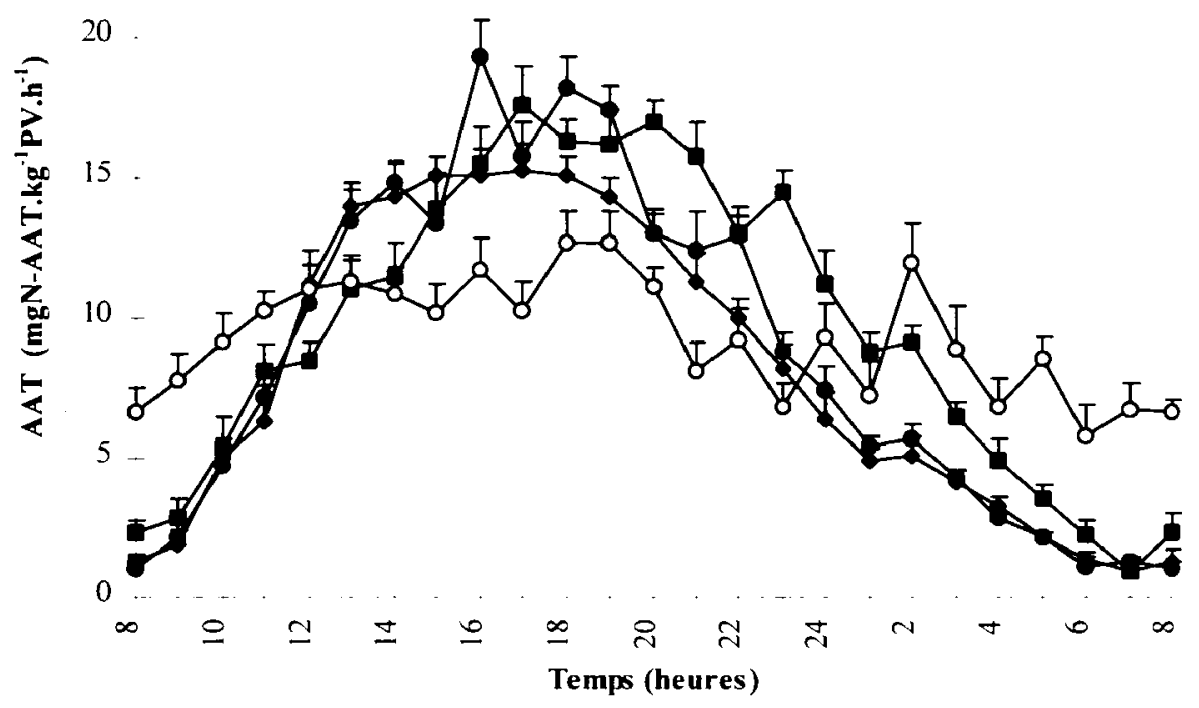

25

(b)

20

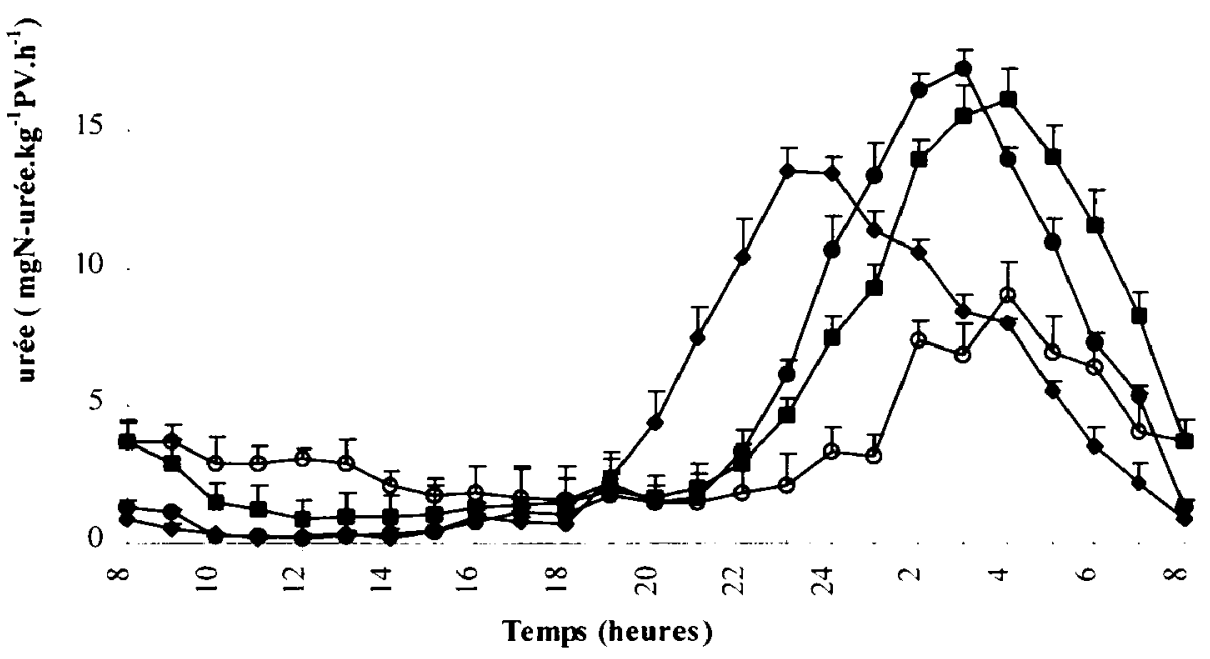

Figure 2

Profil d'excrétion d'azote ammoniacal total (a) et d'azote uréique (b) chez des juvéniles de turbot pour 4 photopériodes : 8L:160 (४), 12L:120(๑), 16L:80 (ם) et 24L:0O (O). La durée du repas correspond à la longueur de la photophase moins 2 heures, l'aliment étant distribué à partir de $9 \mathrm{~h} 00$ pour toutes les conditions expérimentales.

Figure 2

Daily pattern of total ammonia nitrogen (a) and urea (b) excretion for photoperiods : 8L:16D ( $\bullet), 12 \mathrm{~L}: 12 \mathrm{D}(0), 16 \mathrm{~L}: 8 \mathrm{D}(\square)$ et 24L:OD (O). Feeding time was equal to day-length minus 2 hours. First feeding was at 9.00 in the morning for all experimental conditions. 
La plupart des espèces de poissons présentent une activité alimentaire diurne et il existe une relation positive entre la longueur de la photophase et la quantité d'aliment ingéré (BRETT, 1979). Dans notre expérimentation, la durée de l'alimentation étant réglée sur la longueur de la photophase (moins deux heures) et l'aliment apporté ad libitum, la prise alimentaire journalière des turbots n'apparaissait pas dépendante de cette durée. Au contraire, chez d'autres espèces (saumon atlantique par exemple), il existe une relation positive entre la quantité d'aliment ingéré et la longueur de la photophase (THORPE et al., 1988). II semblerait qu'une distribution de la nourriture sur une période minimale de 6 heures (photopériode 8L:160) soit amplement suffisante pour couvrir les besoins quantitatifs journaliers des turbots juvéniles. En effet, si la durée de la photophase est susceptible d'influer sur la quantité d'aliment ingéré, d'autres facteurs tels que la capacité stomacale ou encore la vitesse de transit intestinal conditionnent également la quantité d'aliment consommé quotidiennement par les poissons.

Les hormones thyroïdiennes ayant été proposées comme indicateurs de croissance (BOEUF, 1993), la diminution du niveau plasmatique de $T_{3}$ observée lors du dernier prélèvement pour les photophases courtes pourrait indiquer une baisse du potentiel de croissance. II aurait toutefois fallu poursuivre l'expérience plus longtemps pour le confirmer.

Chez le turbot, comme chez la majorité des téléostéens, l'Azote Ammoniacal Total (AAT) est la principale forme d'excrétion azotée (HANDY et POXTON, 1994). Dans cette étude, il représente environ $75 \%$ de l'excrétion azotée totale mesurée (urée + AAT), valeur comparable avec les travaux de DOSDAT et al. (1995b). Les quantités d'AAT excrétées quotidiennement sont comprises entre 200 et $230 \mathrm{mg}^{\mathrm{kg}}{ }^{-1}$.jour ${ }^{-1}$ et du même ordre de grandeur que celles obtenues chez d'autres poissons plats tels que la plie japonaise, Paralichthys olivaceus (KIKUCHI et al., 1991) et le flétan, Hippoglossus hippoglossus (DAVENPORT, 1990). Au cours du nycthémère, l'excrétion d'AAT présente des variations cycliques, en étroite relation avec la prise alimentaire, et un pic post-prandial est observé. Ces résultats confirment les travaux de DOSDAT et al. (1995b) et BUREL et al. (1996) sur la même espèce. De plus, les profils d'excrétion d'AAT réalisés montrent que pour les photopériodes $8 \mathrm{~L}: 16 \mathrm{O}, 12 \mathrm{~L}: 12 \mathrm{O}, 16 \mathrm{~L}: 8 \mathrm{O}$, la durée du pic post-prandial d'excrétion d'AAT est en relation avec la longueur du repas. Des niveaux élevés sont observés tant que la nourriture est distribuée et le retour à un niveau de base est progressif. Par contre, lorsque les animaux sont soumis à un éclairage continu (alimentation sur 22 heures), une relative stabilité des taux horaires d'excrétion est observée. Nos travaux confirment également que l'excrétion d'urée passe par un maximum en phase nocturne (DOSDAT et al., 1995b ; BUREL et al., 1996). En l'absence de phase obscure, une augmentation des taux horaires d'excrétion d'urée, d'amplitude plus faible, mais correspondant cependant à la phase obscure chez les animaux soumis à une alternance jour-nuit est observée : existerait-il un rythme endogène, calqué sur la photopériode, qui n'aurait pas encore disparu chez ces poissons stabulés deux mois dans ces conditions? Des données récentes obtenues chez certains poissons téléostéens (brochet, Esox lucius ; poisson-zèbre, Brachydanio rerio ; daurade, Sparus aurata ; carassin, Carassius auratus) démontrent la présence de photorécepteurs dans la glande pinéale, contenant un pigment photosensible, "traducteur de l'effet lumière " et représentant une horloge (oscillateur circadien endogène) (BOLLIET et al., 1996), la mélatonine intervenant comme un " donneur de temps interne " (Zeitgeber) de l'organisme (FALCON et al., 1992). Au vu du rythme d'excrétion de l'urée, maintenu chez les turbots en éclairage constant, on peut très probablement imaginer qu'ils fonctionnent comme ces quatre espèces de téléostéen.

\section{CONCLUSION}

Le turbot apparaît comme une espèce marine de téléostéen tempéré qui trouve la meilleure expression de sa croissance vers $17-19^{\circ} \mathrm{C}$ (BUREL et al., 1996) et qui s'adapte 
très bien aux salinités intermédiaires (10-19 ppm, GAUMET et al., 1995). Comme d'autres poissons plats, il apparaît très peu réceptif à la longueur de la photophase. L'habitat naturel du turbot en fait une espèce benthique, vivant, en fonction de son stade de développement, dans des fonds entre 20 et $200 \mathrm{~m}$ et donc en conditions, même saisonnières, assez stables. Cependant, en élevage il se prête parfaitement à des situations différentes. Ces expériences consistant à faire varier un facteur du milieu à chaque fois (monofactorielles) sont certes d'un certain intérêt pour apporter des éléments de physiologie, mais il ne faut surtout pas oublier qu'une situation naturelle ou un élevage représentent la résultante de nombreux facteurs qui varient en même temps au cours du cycle nycthémèral ou des saisons, ou encore avec le pouvoir migrateur des poissons. Aussi, à terme, faudra-t-il passer à des expérimentations plus complexes durant lesquelles plusieurs facteurs pourront varier en même temps.

\section{REMERCIEMENTS}

Nous tenons à remercier I'IFREMER pour son aide, dans le cadre du GDR/PR "Régulation de la croissance chez les poissons d'intérêt aquacole ", qui nous a permis de mener à bien ces expérimentations. Par ailleurs, les auteurs remercient Bruno PETTON pour sa précieuse aide technique, l'entretien des dispositifs expérimentaux et la mise à disposition de juvéniles de qualité.

\section{BIBLIOGRAPHIE}

ANONYME, 1984. In Official methods of analysis of the Association of Official Analytical Chemists, WILLIAMS S. (ed.), AOAC, Arlington, WA, $1141 \mathrm{p}$.

BOEHLERT G.W., 1981. The effects of photoperiod and temperature on laboratory growth of juvenile Sebastes diploproa and a comparison with growth in the field. Fish. Bull., 79, 789-794.

BOEUF G., LE BAIL P.Y., PRUNET P., 1989. Growth hormone and thyroid hormones during Atlantic salmon, Salmo salar L., smolting and transfer to seawater. Aquaculture, 82, 257-268.

BOEUF G., 1993. Salmonid smolting : a pre-adaptation to the oceanic environment. In Fish Ecophysiology, RANKIN J.C. and JENSEN F.B. (eds), Chapman and Hall publishers, 105-135.

BOEUF G., LE BAIL P.Y., 1998. Does light have an influence on fish growth ? Aquaculture, sous presse.

BOLLIET V., BEGAY V., TARAGNAT C., RAVAULT J.P., COLLIN J.P., FALCON J., 1996. Photoreceptor cells of the pike, pineal organ as cellular circadian oscillators. Eur. $J$. Neuroscience, 9, 643-653.

BRETT J.R., 1979. Environmental factors and growth. In Fish physiology, HOAR W.S., RANDALL D.J. and BRETT J.R. (eds), Academic press, New York, 8, 599-675.

BUREL C., PERSON-Le RUYET J., GAUMET F., LE ROUX A., SEVERE A., BOEUF G., 1996. Effects of temperature on growth and metabolism in juvenile turbot. J. Fish Biol., 49, 678-692.

DAVENPORT J., KJORSVIK E., HAUG T., 1990. Appetite, gut transit, oxygen uptake and nitrogen excretion in captive halibut, Hippoglossus hippoglossus L., and lemon sole, Microstomus kitt (Walbaum). Aquaculture, 90, 267-277.

DOSDAT A., GAUMET F., CHARTOIS H., 1995a. Marine aquaculture effluent monitoring : methodological approach to the evaluation of nitrogen and phosphorus excretion by fish. Aqua. Eng., 14, 59-84. 
DOSDAt A., METAILlER R., teTU N., SERVAIS F., CHARTOIS H., HUELVAN C., DESBRUYERES E., 1995b. Nitrogenous excretion in juvenile turbot, Scophthalmus maximus (L.), under controlled conditions. Aquacult. Res., 26, 639-650.

FALCON J., THIBAULT C., BEGAY V., ZACHMANN A., COLLIN J.P., 1992. Regulation of the rhythmic melatonin secretion by fish pineal photoreceptor cells. In Rhythms in fishes, ALI M.A. (ed)., Plenum Press, New York, 167-198

FONDS M., 1979. A seasonal fluctuation in growth rate of young plaice (Pleuronectes platessa) and sole (Solea solea) in the laboratory at constant temperatures and a natural daylight cycle. In Cyclic phenomena in marine plants and animals, NAYLOR E. and R.G. (eds), Proc. 13th Eur. Mar. Biol. Symp., 151-156.

FORSBERG O.I., 1995. Empirical investigations on growth of post-smolt Atlantic salmon (Salmo salar L.) in land-based farms. Evidence of a photoperiodic influence. Aquaculture, 133, 235-248.

GAUMET F., BOEUF G., LE ROUX A., MAYER-GOSTAN N., 1995. Effects of salinity on the ionic balance and growth of juvenile turbot (Scophthalmus maximus). J. Fish Biol., 47, 865-876.

GROSS W.L., ROELOFS E.W., FROMM P.O., 1965. Influence of photoperiod on growth of green sunfish Lepomis cyanellus. J. Fish. Res. Board Can., 22, 1379-1386.

HALLARÅKER H., FOLKVORD A., STEFANSSON S.O., 1995. Growth of juvenile halibut (Hippoglossus hippoglossus) related to temperature, day-length and feeeding regime. Neth. J. Sea Res., 34, 139-147.

HANDY R.D., POXTON M.G., 1994. Nitrogen pollution in mariculture : toxicity and excretion of nitrogenous compounds by marine fish. Fish Biol. Fish., 3, 205-241.

IGLESIAS J., OLMEDO M., OTERO J.J., PELETEIRO J.B., SOLORZANO M.R., 1987. Growth under laboratory conditions of turbot, Scophthalmus maximus, from the ria de Vigo (North-West Spain). Mar. Biol., 96, 11-17.

IMSLAND A., FOLKVORD A., STEFANSSON S.O., 1995. Growth, oxygen consumption and activity of juvenile turbot (Scophthalmus maximus L.) reared under different temperatures and photoperiods. Neth. J. Sea Res., 34, 149-159.

JONES A., BROWN J.A.G., DOUGLAS M.T., THOMPSON S.J., WHITFIELD R.J., 1981. Progress towards developing methods for the intensive farming of turbot (Scophthalmus maximus L.) in cooling water from a nuclear power station. In Aquaculture in heated effluents and recirculation systems, TIEWS K. (ed.), 2, Heinemann, Berlin, 481-496.

KIKUCHI K., TADEKA S., HONDA H., KIYONO M., 1991. Effects of feeding on nitrogenous excretion of Japanese flounder, Paralichthys olivaceus. Nippon Suisan Gakkaishi, 57, 2059-2064.

KÜHLMANN D., QUANTZ G., 1980. Some effects of temperature and salinity on the embryonic development and incubation time in turbot, Scophthalmus maximus L., from the Baltic sea. Meeresforsch., 28(2), 172-178.

MARTINEZ I., DREYER B., AGERSBORG A., LE ROUX A., BOEUF G., 1995. Effects of $\mathrm{T}_{3}$ and rearing temperature on growth and skeletal myosin heavy chain isoform transition during early development in the salmonid Salvelinus alpinus (L.). Comp. Biochem. Physiol., 112B (4), 717-725.

PERSON-Le RUYET J., BAUDIN-LAURENCIN F., DEVAUCHELLE N., METAILLER R., NICOLAS J.L., ROBIN J., GUILLAUME J., 1991. Culture of turbot (Scophthalmus maximus). In Handbook of mariculture, Vol. II., Finfish aquaculture, McVEY J.P. (ed.), CRC Press Boston, 21-41.

SAUNDERS R.L., SPECKER J.L., KOMOURDJIAN M.P., 1989. Effects of photoperiod on growth and smolting in juvenile Atlantic salmon (Salmo salar). Aquaculture, 82, 103117. 
SILVA-GARCIA A.J., 1996. Growth of juvenile gilthead seabream (Sparus aurata L.) reared under different photoperiod regimes. Isr. J. Aquacult.-Bamidgeh, 48, 84-93.

THORPE J.E., MORGAN R.I.G., PRETSWELL D., HIGGINS P.J., 1988. Movements rhythms in juvenile Atlantic salmon, Salmo salar L. J. Fish Biol., 33, 931-940.

WALLER U., 1992. Factors influencing routine oxygen consumption in turbot, Scophthalmus maximus. J. Appl. Ichthyol., 8, 62-71. 